\title{
Propagation of Spin Information at the Supramolecular Scale through Heteroaromatic Linkers
}

\author{
V. Bellini, ${ }^{1, *}$ G. Lorusso, ${ }^{1}$ A. Candini,,${ }^{1}$ W. Wernsdorfer, ${ }^{2}$ T. B. Faust,${ }^{3}$ G. A. Timco, ${ }^{3}$ \\ R.E.P. Winpenny, ${ }^{3,4}$ and M. Affronte ${ }^{5,1}$ \\ ${ }^{1}$ S3-Institute Nanoscience-CNR, Via Campi 213/A, 41125 Modena, Italy \\ ${ }^{2}$ Laboratoire Louis Néel, avenue des Martyres 24, Grenoble, France \\ ${ }^{3}$ School of Chemistry, The University of Manchester, Oxford Road, Manchester, M13 9PL, United Kingdom \\ ${ }^{4}$ The Photon Science Institute, The University of Manchester, Oxford Road, Manchester, M13 9PL, United Kingdom \\ ${ }^{5}$ Dipartimento di Fisica, Universitá di Modena e Reggio Emilia, Via Campi 213/A, 41125 Modena, Italy
}

(Received 28 February 2011; published 3 June 2011)

\begin{abstract}
We report an in-depth study on how spin information propagates at supramolecular scale through a family of heteroaromatic linkers. By density-functional theory calculations, we rationalize the behavior of a series of $\mathrm{Cr}_{7} \mathrm{Ni}$ dimers for which we are able to systematically change the aromatic linker thus tuning the strength of the magnetic interaction, as experimentally shown by low temperature micro-SQUID and specific heat measurements. We also predict a $\cos ^{2}$ dependence of the magnetic coupling on the twisting angle between the aromatic cycles in bicyclic linkers, a mechanism parallel to charge transport on similar systems [L. Venkataraman et al., Nature (London) 442, 904 (2006)].
\end{abstract}

DOI: 10.1103/PhysRevLett.106.227205

PACS numbers: 75.50.Xx, 71.15.Mb, 75.30.Et

Controlling and understanding the propagation of spin information at the molecular scale is an intriguing issue involving both fundamental sciences and the possibility of designing molecular devices of interest for information technologies. In order to make two magnetic units weakly interact at supramolecular (nanometer) scale, local through-bond interactions are preferable to dipolar interactions, as the latter has essentially long-range character and is difficult to tune without large structural modification in the systems. While exchange interactions, stemming from direct overlapping of nearest neighboring metal orbitals, as well as superexchange, mediated by the orbitals of bridging, e.g., oxygen, atoms, are mechanisms which have been studied in detail both experimentally and theoretically, spin interactions between metal centers through long organic groups still remain largely unexploited. Aromatic organic linkers are suitable candidates to create local interactions and have been studied in the 1980s and 1990s within the quest for room temperature metallorganic magnets. Molecular dimers, where the magnetic parts of the single units are represented by single paramagnetic ions, have been investigated in this context. The strength of the interaction, electronic and magnetic, between the units linked by an aromatic bridge, has been found to obey some general empirical rules [1]: (1) the larger the number of bonds which compose the interaction path, the smaller the interaction is; (2) charge and spin polarization induced by bonding to a metal site proceed in an alternating fashion in aromatic cycles; (3) since a magnetic interaction can be sustained by different bond paths in the linker, the strength of the interaction should depend on whether quantum constructive or destructive interference between paths with different lengths arise [2]. These findings have been discussed so far in terms of simplified model orbital methods [3-5], and only more recently refined densityfunctional characterizations of dimeric complexes appeared in the literature [6-8]. The next challenge is to couple magnetically two (or more) complex molecules without perturbing the properties of the individual units. This allows the entanglement of spins of molecular units [9] and eventually the encoding of two-qubits gates or the communication of magnetic information at supramolecular (nanometer) scale [10]. Supramolecular dimers of single molecule magnets, which involve linking $\mathrm{Mn}_{4}$ cages into dimers through $\mathrm{C}-\mathrm{H} \cdots \mathrm{Cl}$ hydrogen bonds, have been reported [11]. Although magnetic interaction is achieved [12], tuning this interaction is not straightforward.

Heterometallic $\mathrm{Cr}_{7} \mathrm{Ni}$ molecular rings combine special chemical and magnetic features that allow us to define a strategy for linking molecular magnetic units. The key ingredient is the presence-within the $\mathrm{Cr}^{\mathrm{III}}\left(S_{\mathrm{Cr}}=3 / 2\right)$ wheel-of an "extra" divalent ion $\left(\mathrm{Ni}^{\mathrm{II}}, S_{\mathrm{Ni}}=1\right)$ that allows us to selectively build both the chemical and the magnetic link between molecular units. A series of $\mathrm{Cr}_{7} \mathrm{Ni}$ dimers have been recently synthesized, with the general formula $\left[\left\{\mathrm{Cr}_{7} \mathrm{NiF}_{3}(\mathrm{Etglu})\left(\mathrm{O}_{2} \mathrm{C}^{t} \mathrm{Bu}\right)_{16}\right\}_{2}(\mathrm{~L})\right]$ with $\mathrm{L}$ being the heteroaromatic linker [13]. We present in the following a thorough study of five different dimers, with $L=$ pyrazine (pyr, in short), bidimethylpyrazolyl (bipz), 4,4'-bipyridyl (bipy), trans-1,2-bipyridylethene (bipyet), and bipyridyltetrazine (bipytz) (see Fig. 1 for a sketch of the molecular structures of these dimers, and EPAPS [14] for more informations). The first linker (pyr) is the only one composed of a single heterocycle, the second (bipz) is the only one built with two five-membered rings (pyrazole), the third and fourth (bipy- and bipyet-) are essentially made of two pyridine rings, while the last (bipytz) contain three aromatic rings (two pyridine and one 


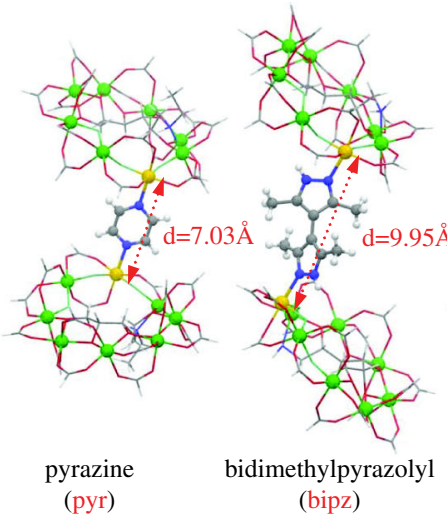

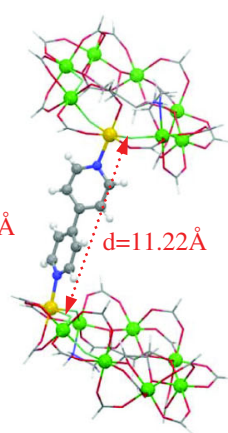

bipyridyl

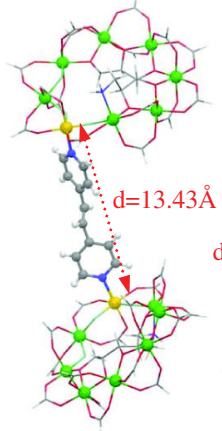

bipyridylethene (bipyet)

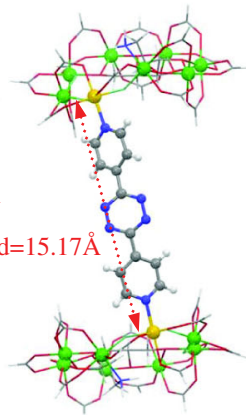

bipyridyltetrazine (bipytz)

FIG. 1 (color online). The molecular structures of pyr, bipz, bipy, bipyet, and bipytz $\mathrm{Cr}_{7} \mathrm{Ni}$ purple rings. The atoms of the linkers (N, blue; $\mathrm{C}$, gray; $H$, white) and the metal ions in the rings ( $\mathrm{Ni}$, yellow; $\mathrm{Cr}$, green) have been highlighted; the inter-rings through-space $\mathrm{Ni}-\mathrm{Ni}$ distances (d) are also given for each dimer.

tetrazine). Ni-Ni interring distances range from 7 to more than $15 \AA$, as reported in Fig. 1. Dihedral angles between the cycles, as measured by x-ray diffraction, are around $41^{\circ}$ for bipy, $56^{\circ}$ for bipz, and $28^{\circ} / 32^{\circ}$ for bipytz, while for bipyet no clear angle could be defined due to the ethene block. The dimers are found to pack into different crystal structures, with negligible interdimer magnetic interactions. The intraring magnetic coupling is of the antiferromagnetic type, leading to a molecular $S=1 / 2$ Kramer ground state doublet for all the $\mathrm{Cr}_{7} \mathrm{Ni}$ rings. The dimers depicted in Fig. 1 are only some representative derivatives of a larger family of dimers, which can be synthesized with relative ease [15].

We first address the theoretical characterization of the systems. Calculations have been performed by the densityfunctional theory (DFT) module of the NWCHEM quantum chemistry package [16]. The full systems (rings and bridge) have been taken into account with some pruning of the organic ligands surrounding the magnetic cores of the rings has been performed (more information on the theoretical method could be found in Sec. I in the supplemental material [14]). $p$ electrons of the linkers are distributed over two types of orbitals, the ones that occur in localized covalent bonds (with label $\sigma$ ) and the ones that occupy delocalized bonds ( $\pi$ electrons). Conjugation due to $\pi$ orbitals is a good candidate to drive magnetic interactions at $\mathrm{nm}$ scale. The magnetic interaction is maximum when overlap (both in space and in energy) between the spin-polarized frontier orbitals of the magnetic centers, in our case $\mathrm{Ni}$, and orbitals of the linker anchoring atom $\mathrm{N}$ is found. $\mathrm{Ni}^{\mathrm{II}}$ ions, have nominally a $3 d^{8}$ electronic configurations; in an nearly orthorhombic environment only $d$ orbitals with $E g$-like symmetry are spin polarized. As the local axis around the Ni atoms are rotated from the plane of the heterocycles in the linkers, and some deviation from orthorhombicity is found for the coordination shell of $\mathrm{Ni}$ atoms, both $\sigma$ and $\pi$ orbital of the extended molecule can in principle favor magnetic interactions between the spin of the two rings. In Fig. 2 we plot the spin-polarized electron density isosurfaces for a very low isovalue $\left(= \pm 0.0005\right.$ electrons/a.u. $\left.{ }^{3}\right)$, and zoom onto the relevant Ni-linker-Ni coupling region. Several observations can be made. The first is that in all the linkers, an alternation of spin polarization when moving from one atom to the next, following bond paths, is present in the linkers, and this polarization involves primarily orbitals with $\pi$ character. Polarization of $\sigma$ orbitals in the linkers, although present on the $\mathrm{N}$ atoms bonded to $\mathrm{Ni}$, fades away within few bonds,

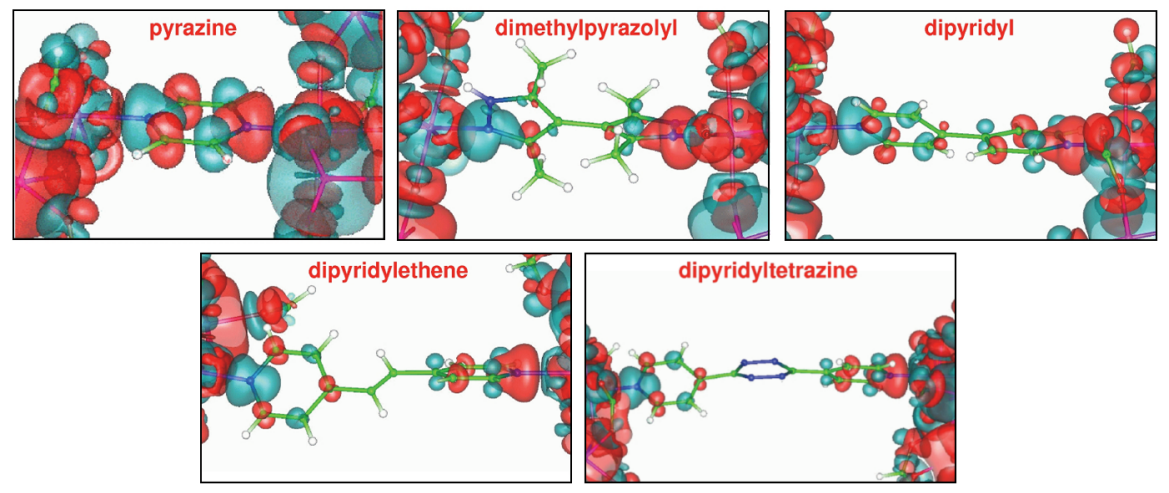

FIG. 2 (color online). Spin-polarization density in pyr, bipz, bipy, bipyet, and bipytz dimers, zoomed in the region of the linker, for isovalues of + (cyan) and - (red) 0.0005 electrons $/$ a.u. ${ }^{3}$ 
and vanishes altogether for inner $\mathrm{C}$ atoms. Because of the spin alternation rule, whether ferromagnetic or antiferromagnetic interactions between magnetic centers is seen depends on the number of $\mathrm{C} / \mathrm{N}$ atoms in the bonding pathway; if an even number of $\mathrm{C} / \mathrm{N}$ atoms is present the exchange is antiferromagnetic, while if an odd number of $\mathrm{C} / \mathrm{N}$ atoms is present ferromagnetic coupling should be encountered. Exchange between the two $\mathrm{Ni}$ atoms can follow two different possible paths around the six- and five-membered rings; in bipz, there exist pathways with both odd and even numbers of $\mathrm{C} / \mathrm{N}$ atoms, supporting simultaneously ferro- or antiferromagnetic exchange, respectively. Destructive interference between these two paths leads to a very low spin polarization of the orbitals in the inner region of the bipz, despite them being closer than in the other bicyclic dimers. Delocalization of the $\pi$ system orbitals also becomes less efficient as the number of bonds increase; consequently the spin polarization in the inner region of the linker is larger for pyr than for bipy or bipyet; clearly if we increase the distance between the $\mathrm{Ni}$ atoms, spin polarization reduced still further, that being the case of bipytz.

In order to provide quantitative information, we have performed total energy calculations of broken-symmetry (BS) spin configurations, and mapped them onto the microscopic spin Hamiltonian of the type

$$
H=2 J^{\prime} \mathbf{S}_{\mathrm{Ni} 1} \cdot \mathbf{S}_{\mathrm{Ni} 2}
$$

where $J^{\prime}$ is the isotropic exchange between the Ni spin moments, and $\mathbf{S}_{\mathrm{Ni} 1}$ and $\mathbf{S}_{\mathrm{Ni} 2}$ are the spin moments of the two Ni ions $\left(S_{\mathrm{Ni} 1}=S_{\mathrm{Ni} 2}=1\right)$. Intraring magnetic interactions between $\mathrm{Cr}-\mathrm{Cr}$ and $\mathrm{Cr}-\mathrm{Ni}$ spins are of the antiferromagnetic (AFM) type, leading to a $S=1 / 2$ ground state for the single $\mathrm{Cr}_{7} \mathrm{Ni}$ rings. While retaining this AFM states for both the rings, we have characterized the two situations where the $S=1 / 2 \mathrm{Cr}_{7} \mathrm{Ni}$ spins are either coupled ferromagnetic (FM) or AFM to each other. In terms of the microscopic coupling between the two $S=1 \mathrm{Ni}$ spins, this leads to two states with energies $E_{\mathrm{FM}}=2 J^{\prime}$ and $E_{\mathrm{AFM}}=-2 J^{\prime} ; J^{\prime}$ is then simply given by $1 / 4$ of the total energy difference between the FM and AFM states of the dimer. The calculated $J^{\prime}$ values are $367,8,48,56$, and $22 \mu \mathrm{eV}$ for pyr, bipz, bipy, bipyet, and bipytz, respectively (see supplemental information [14] for more details). These are tiny interactions that are near the numerical precision of the DFT method; yet if we consider the trend, the calculations show similar $J^{\prime}$ for bipy, bipyet, a value 7 times larger for pyr, and smaller values for bipz and bipytz.

This findings are fully supported by experiments. From the magnetic point of view, the family of $\mathrm{Cr}_{7} \mathrm{Ni}$ rings behave, at low temperatures, as ideal two-level mesoscopic systems with an effective $S=1 / 2$ and higher multiplets that are relevant at high temperature $(T)$ /applied magnetic fields $(H)$ or for effects due to the mixing of magnetic states $[17,18]$. Low temperature specific heat $C(T, H)$ data at $3 \mathrm{~T}$ (Fig. $1 \mathrm{~S}$ in the supplemental material [14]) demonstrates that the pattern of the lowest lying states of the single $\mathrm{Cr}_{7} \mathrm{Ni}$ rings are preserved also in the supramolecular systems. To reveal the presence of the interring interaction we have performed micro-SQUID measurements at $40 \mathrm{mK}$. The resulting magnetization curves are plotted in Fig. 3. Data are normalized to the saturation value $M_{S}$ and for simplicity only the part of the hysteresis loop with increasing field is plotted. In order to enlighten the transition between the singlet and triplet states, the magnetization cycle is magnified between $H= \pm 0.1 T$, whereas for all the dimers, $M(H)$ saturates above $1 \mathrm{~T}$ (for the complete hysteresis loops see Fig. $2 \mathrm{~S}$ in the supplemental material [14]). If the $S_{\text {tot }}=0$ state is the ground state in zero field, we should be able to see a spin-state crossing by increasing the field, with one component of the paramagnetic $S_{\text {tot }}=1$ state falling in energy as field increases (visible as an inflection point of the magnetization curve). The crossing point in the magnetization, i.e., the gap between inflection points, can then be related to the energy gap between the $S_{\text {tot }}=0$ and $S_{\text {tot }}=1$ states [9]. We can see in Fig. 3 that the level crossings occur at $B=$ $\pm 0.05 T$ for bipy and at $B= \pm 0.04 T$ for bipyet, while it appears at $B= \pm 0.30 T$ for pyr. Fitting this crossing to the microscopic Hamiltonian equation (1), we obtain $J^{\prime} / k_{B}=$ $1.0 \mathrm{~K}$ for the pyr-linked dimers, approximately 6 times the value for the bipy dimer, where $J^{\prime} / k_{B}=0.16 \mathrm{~K}$ [9], and $\sim 20 \%$ weaker for the bipyet-linked one (positive values refer to antiferromagnetic interaction). For the other two compounds (bipytz and bipz), the magnetic coupling is too small, and no inflection points are visible in the magnetization curves. We can thus conclude that in bipytz and bipz derivatives the interaction between the two $\mathrm{Cr}_{7} \mathrm{Ni}$ rings must be weaker than $100 \mathrm{mK}$. These data are in excellent agreement with the trend among the different dimers evidenced in the DFT analysis reported above.

It is now evident that the length of the linker is not the only attribute one has to consider when seeking a tailored spin coupling. As a matter of fact, the conformation of the

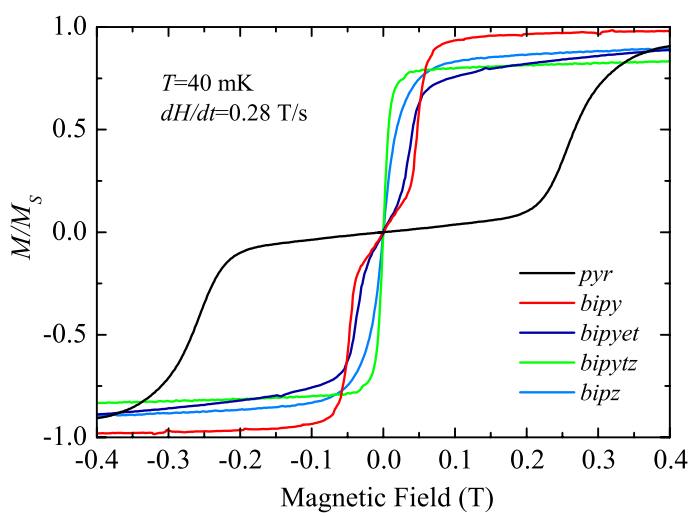

FIG. 3 (color online). Micro-SQUID magnetization $M(H)$ vs magnetic field $H$ for the pyr, bipz, bipy, bipyet, and bipytz dimers. The experiments were performed at $T=40 \mathrm{mK}$, using sweeping rate $d H / d t=0.28 \mathrm{~T} / \mathrm{s}$. Data are normalized to the saturation value $M_{S}$. 


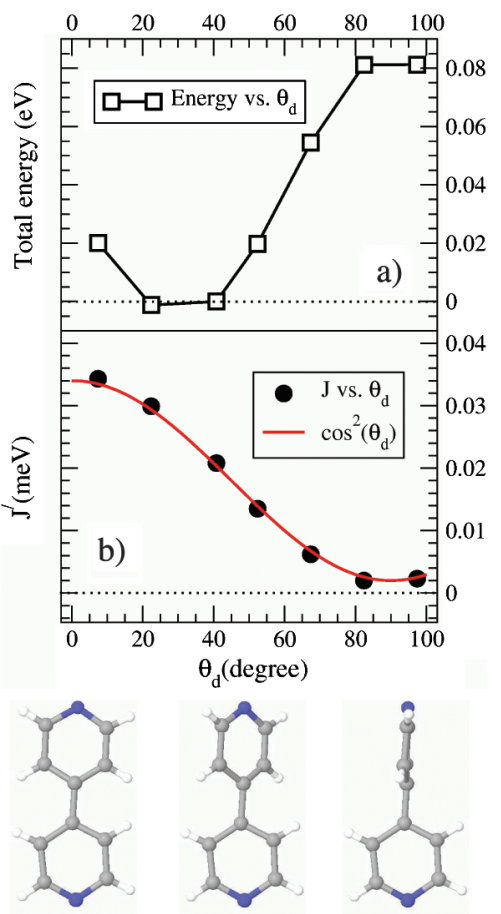

FIG. 4 (color online). (a) Total energy and b) exchange interaction parameter $J^{\prime}$ in the bipy-linked dimers, as a function of the dihedral angle $\theta_{d}$ between the two pyridine cycles.

linker, the angles between the two heterocycles, or the angles between the cycles and the $\mathrm{Ni}$ coordination axes do play an important role, modifying the conjugation level and consequently the interring spin coupling. We have thus performed computational experiments for the bipy dimer, by calculating the magnetic interaction as a function of the dihedral angle between the two pyridine cycles; to do this, we have coherently rotated the molecules in the space, keeping fixed the geometry of the Ni-linker contacts, in order to selectively study the dependence of $J$ and total energy as a function of linker conformation only. Total energy calculations indicate a preferred twisted configuration with dihedral angle around $20-30^{\circ}$ (see Fig. 4, upper panel). The variation of $J^{\prime}$ as a function of the dihedral angle $\theta_{d}$ is plotted in Fig. 4, lower panel. Maximum magnetic coupling would be achieved when the two cycles are coplanar, and overlap between $\pi$ orbitals, and consequently conjugation, is at its maximum. As evidenced by the continuous line the variation of $J^{\prime}$ follows closely a $\cos ^{2}\left(\theta_{d}\right)$ curve. Interestingly, the conductance through biphenyl junctions have shown similar $\cos ^{2}\left(\theta_{d}\right)$ behavior $[19,20]$, and this has been traced back to the $\cos \left(\theta_{d}\right)$ dependence of the overlap of the $\pi$ orbitals. [21] We may qualitatively understand the similar angular dependency considering that the spin information extends through the linkers by a spin-polarization mechanism, and in first approximation, the sequential polarization of the $\pi$ orbitals at the $\mathrm{C} / \mathrm{N}$ sites involves hopping of electrons in the conjugated system, as much as molecular electron transfer, which is directly related to the $\pi$ - $\pi$ orbital overlap throughout the linker. Therefore, a very interesting parallelism between charge and spin propagation in bicyclic aromatic linker emerges from these works.

In summary, we have provided an in-depth description of the propagation of spin information between two molecular macrocycles through a family of aromatic linkers that we have considered as paradigmatic case. The numerical observation of a $\cos ^{2}$ dependence on torsion angles is also promising, and paves the way for a whole series of possible experimental investigations, by systematically varying the organic bridges and the magnetic frontier atoms, in order to tune and choose the appropriate coupling regime, switching the interaction on and off at will, by perturbing the organic linker using a mechanical stimulus.

This work has been partially supported by FP7-ICT FET Open MolSpinQIP project, Contract No. 211284, by the EPSRC (U.K.), and from MIUR-PRIN projects (I), Contracts No. 2008PARRTS and No. 2008NX9Y7. We acknowledge the CINECA supercomputing center for the availability of high performance computing resources and support.

*valerio.bellini@unimore.it

[1] D. E. Richardson and H. Taube, J. Am. Chem. Soc. 105, 40 (1983).

[2] V. Marvaud, J.-P. Launay, and C. Joachim, Chem. Phys. 177, 23 (1993).

[3] J. A. McCleverty and M. D. Ward, Acc. Chem. Res. 31, 842 (1998).

[4] H. M. McConnell, J. Chem. Phys. 39, 1910 (1963).

[5] H. C. Longuet-Higgins, J. Chem. Phys. 18, 265 (1950).

[6] A. Bencini et al., J. Phys. Chem. A 102, 10545 (1998).

[7] F. Nunzi et al., J. Phys. Chem. C 111, 618 (2007).

[8] J. Ferrando-Soria et al., Inorg. Chim. Acta 363, 1666 (2010).

[9] A. Candini et al., Phys. Rev. Lett. 104, 037203 (2010).

[10] F. Troiani and M. Affronte, Chem. Soc. Rev. (in press).

[11] D. N. Hendrickson et al., J. Am. Chem. Soc. 114, 2455 (1992).

[12] W. Wernsdorfer et al., Nature (London) 416, 406 (2002).

[13] G. A. Timco et al., Angew. Chem., Int. Ed. 47, 9681 (2008).

[14] See supplemental material at http://link.aps.org/ supplemental/10.1103/PhysRevLett.106.227205 for supplementary information.

[15] T. B. Faust et al. (to be published).

[16] E.J. Bylaska et al., NWChem, A Computational Chemistry Package for Parallel Computers, Version 5.1, Pacific Northwest National Laboratory, Richland, Washington, USA, 99352-0999, 2007.

[17] M. Affronte et al., Phys. Rev. B 68, 104403 (2003).

[18] S. Carretta et al., Phys. Rev. B 72, 060403(R) (2005).

[19] L. Venkataraman et al., Nature (London) 442, 904 (2006).

[20] M.S. Hybertsen et al., J. Phys. Condens. Matter 20, 374115 (2008).

[21] S. Woitellier, J.-P. Launay, and C. Joachim, Chem. Phys. 131, 481 (1989). 